\title{
Turbulent heat transport regimes in a channel
}

\author{
B. Castaing* \\ Laboratoire des écoulements géophysiques et industriels \\ Domaine Universitaire, CS 40700, 38058 Grenoble Cedex 9, France \\ E. Rusaouën, J. Salort, and F. Chillà ${ }^{\dagger}$ \\ Univ Lyon, Ens de Lyon, Univ Claude Bernard, \\ CNRS, Laboratoire de Physique, F-69342 Lyon, France
}

(Dated: November 4, 2016)

\begin{abstract}
In this paper we focus on the turbulent transport of a scalar through a channel. The scalar flux, and the corresponding scalar concentration gradient along the channel allow to define a Nusselt number and a Grashof number. While the relation between the large scale velocity field and the input energy rate show a perfect inertial (turbulent) behavior, three different regimes can be distinguished, with different scalings between Nusselt and Grashof numbers.
\end{abstract}

\section{INTRODUCTION}

In the past decade, scalar free convection in a tiltable channel appeared as an interesting model flow for evidencing the base mechanisms of convection [1-11]. Different regimes have been evidenced depending on the angle $\psi$ between the channel axis and the vertical one, the Prandtl (or Schmidt) number $\nu / \kappa$, or the amplitude of density differences. $\nu$ is the kinematic viscosity of the fluid, and $\kappa$, the diffusion coefficient of the active scalar.

The typical geometry is schematized in figure $1 . \mathrm{A}$ channel connects two chambers. The chambers have different temperatures, in the case of heat transport, different scalar concentrations in the other cases. For the sake of clarity, we shall concentrate on heat transport, speaking of temperature instead of scalar concentration, of heat flux instead of scalar flux, but all what follows can be easily translated in the other language.

The Boussinesq equations write:

$$
\begin{aligned}
\partial_{t} v_{i}+v_{j} \partial_{j} v_{i} & =-\frac{\partial_{i} p}{\rho}-g_{i} \alpha \vartheta+\nu \partial_{j} \partial_{j} v_{i} \\
\partial_{t} \vartheta+v_{j} \partial_{j} \vartheta & =\kappa \partial_{j} \partial_{j} \vartheta \\
\partial_{j} v_{j}=0 &
\end{aligned}
$$

$g$ is the gravitation acceleration, $p$ the pressure and $\rho$ the density of the fluid. $\vartheta$ is the temperature, but could as well be the scalar concentration. The origin of $\vartheta$ is such that its mean value on the channel is zero. $\alpha$ is the coefficient of thermal expansion.

We are interested in the turbulent case, where the mean diffusive heat flux is negligible compared to the convective one. Multiplying equation 1 by $v_{i}$ and averaging, it is easy to show that the kinetic energy input rate per unit mass, $\epsilon$, is simply related to the heat flux $Q_{i}[12]$ :

$$
\epsilon=-g_{i} \alpha\left\langle\vartheta v_{i}\right\rangle=-g_{i} \alpha \frac{Q_{i}}{C_{p}}
$$

\footnotetext{
* bernard.castaing627@laposte.net

† Francesca.Chilla@ens-lyon.fr
}

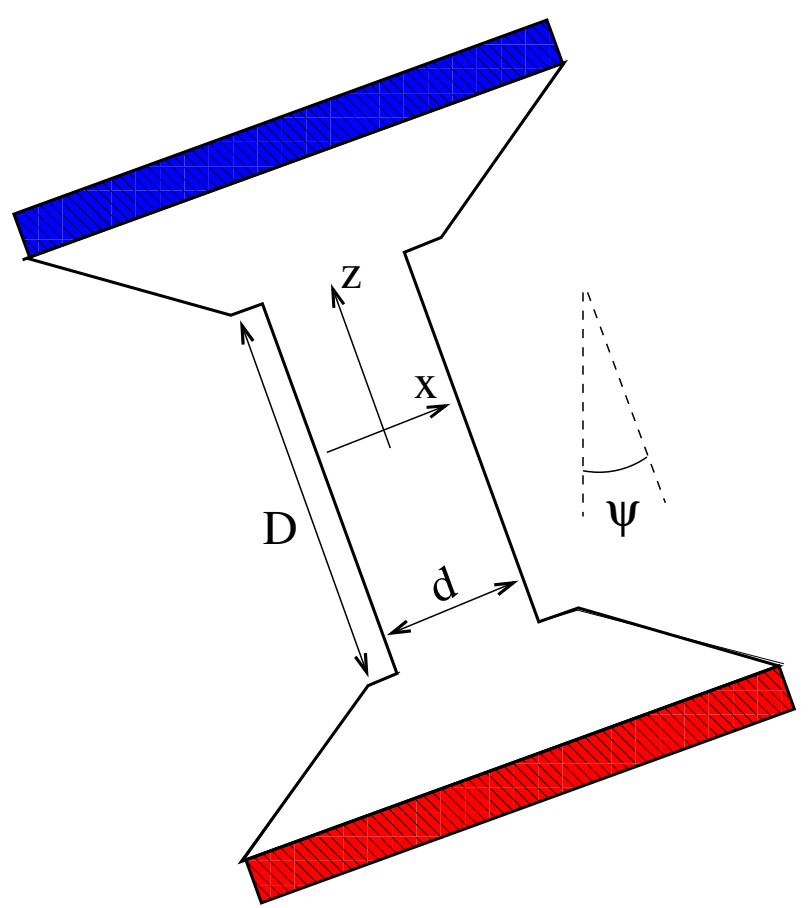

FIG. 1. A sketch of the typical cell showing the definition of the coordinates, and of the inclination angle $\psi$.

where $C_{p}$ is the heat capacity per unit volume. $\epsilon$ can also be expressed as:

$$
\epsilon=\nu\left\langle\partial_{i} v_{j} \partial_{i} v_{j}\right\rangle
$$

which gives a useful evaluation for the typical velocity gradients:

$$
\sqrt{\left\langle\partial_{i} v_{j} \partial_{i} v_{j}\right\rangle}=\left(\frac{\epsilon}{\nu}\right)^{1 / 2}
$$

The characteristics of the inertial regimes is that the velocity, at constant inclination angle, scale with $[6,7]$ :

$$
U_{t}=\left(\frac{Q d g \alpha}{C_{p}}\right)^{1 / 3}
$$


where $d$ is the channel diameter.

In the same regimes, as can be seen from equation 1 , temperature fluctuations scale with $U_{t}^{2}$. In spite of this great unity in the scaling behavior, three different regimes have been observed, concerning the relation between the temperature (or scalar concentration) gradient:

$$
\beta=-\frac{d\langle\vartheta\rangle}{d z}
$$

along the channel, and the heat flux $Q$, thus between the Nusselt number:

$$
N u=\frac{Q}{C_{p} \kappa \beta}
$$

and the Grashof number:

$$
G r=\frac{g \alpha \beta d^{4}}{\nu^{2}}
$$

At large Grashof number, every one [5, 7, 10, 11] agree with:

$$
\frac{N u}{P r} \propto G r^{1 / 2}
$$

At lower Grashof number, Riedinger et al. [8] evidenced a regime:

$$
N u \propto G r^{2}
$$

without precision on the Prandtl number (Pr) dependence. Recently, Pawar and Arakeri [15] observed a new regime:

$$
\frac{N u}{P r} \propto G r^{0.3}
$$

The goal of this paper is to explain these regimes, and to precise their $\mathrm{Pr}$ dependence. We shall show that the $G r^{0.3}$ regime can be intermediate between the two other ones, and disappears for $\operatorname{Pr}<1$. We shall call this regime the Batchelor regime (for reasons which appear below). In a similar way, the Soft Turbulence regime $\left(G r^{2}\right)$ should disappear for $\operatorname{Pr}>>1$. Following Riedinger et al [8], we shall call the $N u \propto G r^{1 / 2}$ regime the Hard Turbulence one. We develop our arguments in the following section.

\section{EVALUATION OF $\beta$}

Our evaluation of $\beta$ will be based on two different expressions for the entropy production per unit volume $\dot{S}$. On the one hand, with a macroscopic point of view,

$$
\dot{S}=-\frac{Q_{i} \partial_{i} T}{T^{2}} \simeq \frac{Q \beta}{T^{2}}
$$

where the absolute temperature $T=T_{o}+\langle\vartheta\rangle$, and $T_{o}$ is the mean temperature of the channel. On the other hand, this entropy production can be related to the microscopic dissipative temperature gradients:

$$
\dot{S}=C_{p} \kappa \frac{\left\langle\partial_{i} \vartheta \partial_{i} \vartheta\right\rangle}{T^{2}}
$$

This gives a formal expression for $\beta$ :

$$
\beta=C_{p} \kappa \frac{\left\langle\partial_{i} \vartheta \partial_{i} \vartheta\right\rangle}{Q}
$$

Calling $\theta=\vartheta-\langle\vartheta\rangle$ the temperature fluctuation, and neglecting the average gradients compared to the instantaneous ones, $\left\langle\partial_{i} \vartheta \partial_{i} \vartheta\right\rangle$ can be evaluated as the mean squared fluctuation at the temperature dissipative scale $\left\langle\theta^{2}\right\rangle_{D}$, divided by this dissipative scale $\eta_{\theta}$ squared:

$$
\left\langle\partial_{i} \theta \partial_{i} \theta\right\rangle \simeq \frac{\left\langle\theta^{2}\right\rangle_{D}}{\eta_{\theta}^{2}}
$$

The evaluation of $\left\langle\theta^{2}\right\rangle_{D}$ and $\eta_{\theta}$ will differ, depending if the Prandtl number is larger or smaller than 1.

\section{A. Small Prandtl}

Let us first assume that the Reynolds number is sufficient to have a Kolmogorov velocity inertial range, between the velocity correlation scale $\ell$ and the velocity dissipation scale $\eta$. At small Prandtl number, the temperature dissipative scale is larger than the velocity one: $\eta_{\theta}>\eta . \eta_{\theta}$ is such that the typical stirring frequency at this scale equals the dissipation frequency:

$$
\frac{v\left(\eta_{\theta}\right)}{\eta_{\theta}} \simeq \frac{U_{t}\left(\eta_{\theta} / \ell\right)^{1 / 3}}{\eta_{\theta}} \simeq \frac{\kappa}{\eta_{\theta}^{2}}
$$

This qualitatively means that, at scales lower than $\eta_{\theta}$, due to the rapidity of diffusion, stirring is unable to enhance the temperature gradients. The temperature dissipative scale $\eta_{\theta}$ can then be expressed as:

$$
\eta_{\theta} \simeq \ell\left(\frac{\kappa}{U_{t} \ell}\right)^{3 / 4}
$$

In a Kolmogorov 41 approach, the temperature fluctuation at the scale $\eta_{\theta}$ writes [13]:

$$
\left\langle\theta^{2}\right\rangle_{D} \simeq\left\langle\theta^{2}\right\rangle\left(\frac{\eta_{\theta}}{\ell}\right)^{2 / 3}
$$

According to equation 1, the mean squared temperature fluctuation $\left\langle\theta^{2}\right\rangle$ in an inertial regime, is of order:

$$
\left\langle\theta^{2}\right\rangle \simeq \frac{U_{t}^{4}}{(g \alpha \ell)^{2}}
$$


Using equation 17, we have thus the following estimate for $\left\langle\partial_{i} \theta \partial_{i} \theta\right\rangle$

$$
\begin{aligned}
\left\langle\partial_{i} \theta \partial_{i} \theta\right\rangle & \simeq \frac{U_{t}^{4}}{(g \alpha \ell)^{2}}\left(\frac{\eta_{\theta}}{\ell}\right)^{2 / 3} \frac{1}{\eta_{\theta}^{2}} \\
& =\frac{U_{t}^{4}}{\left(g \alpha \ell^{2}\right)^{2}}\left(\frac{\eta_{\theta}}{\ell}\right)^{-4 / 3} \\
& =\frac{U_{t}^{4}}{\left(g \alpha \ell^{2}\right)^{2}} \frac{U_{t} \ell}{\kappa}=\frac{U_{t}^{5}}{\kappa(g \alpha)^{2} \ell^{3}}
\end{aligned}
$$

and for $\beta$, using equations 7,16 :

$$
\beta \simeq \frac{\kappa d g \alpha}{U_{t}^{3}} \frac{U_{t}^{5}}{\kappa(g \alpha)^{2} \ell^{3}}=\frac{U_{t}^{2} d}{g \alpha \ell^{3}}
$$

The Nusselt number $N u$ is given by:

$$
\frac{N u}{\operatorname{Pr}}=\frac{Q}{C_{p} \nu \beta}=\frac{U_{t}^{3}}{d g \alpha \nu \beta}
$$

We now can write, using equation 24:

$$
\begin{aligned}
\frac{N u}{P r} & \simeq\left(\frac{\beta g \alpha \ell^{3}}{d}\right)^{3 / 2} \frac{1}{d g \alpha \nu \beta} \\
& =\left(\frac{\ell}{d}\right)^{9 / 2}\left(\frac{g \alpha \beta d^{4}}{\nu^{2}}\right)^{1 / 2}=A G r^{1 / 2}
\end{aligned}
$$

where $A=(\ell / d)^{9 / 2}$ is a constant. We thus find, as expected the Hard Turbulence regime (Small Prandtl Hard Turbulence SPHT).

At lower Reynolds number, the temperature dissipative scale $\eta_{\theta}$ join $\ell$ [13]. We simply have to make $\eta_{\theta}=\ell$ in equation 22 :

$$
\left\langle\partial_{i} \theta \partial_{i} \theta\right\rangle \simeq \frac{U_{t}^{4}}{\left(g \alpha \ell^{2}\right)^{2}}
$$

Then:

$$
\beta \simeq \frac{\kappa d g \alpha}{U_{t}^{3}} \frac{U_{t}^{4}}{\left(g \alpha \ell^{2}\right)^{2}}=\frac{\kappa U_{t} d}{g \alpha \ell^{4}}
$$

and:

$$
\begin{aligned}
\frac{N u}{P r} & \simeq\left(\frac{\beta g \alpha \ell^{4}}{d \kappa}\right)^{3} \frac{1}{d g \alpha \nu \beta} \\
& =\left(\frac{\ell}{d}\right)^{12}\left(\frac{g \alpha \beta d^{4}}{\nu^{2}}\right)^{2}\left(\frac{\nu}{\kappa}\right)^{3}=B P r^{3} G r^{2}
\end{aligned}
$$

where $B=(\ell / d)^{12}$ is a constant. This is the Soft Turbulence scaling (Small Prandtl Soft Turbulence SPST). Note however that it does not coincide here with the absence of inertial range: as $\eta_{\theta}>\eta$, we yet have $\ell>\eta$.

The two scalings above can be presented as:

$$
N u=A\left(G r P r^{2}\right)^{1 / 2} \text { and } N u=B\left(G r P r^{2}\right)^{2}
$$

which shows that in a diagram $N u$ versus $G r P r^{2}$ all the different (small) Prandtl numbers merge on a single curve (at constant inclination angle $\psi$ ).

\section{B. Hight Prandtl}

When the Prandtl number is large, the high Reynolds number situation is different. Indeed, the Kolmogorov inertial range is followed by a Batchelor range of scales [14] in which the temperature fluctuations remain approximately constant. The temperature dissipative scale $\eta_{\theta}$ is now smaller than the velocity one $\eta$ :

$$
\frac{v\left(\eta_{\theta}\right)}{\eta_{\theta}}=\frac{v(\eta)}{\eta} \simeq\left(\frac{\epsilon}{\nu}\right)^{1 / 2}=\left(\frac{U_{t}^{3}}{d \nu}\right)^{1 / 2} \simeq \frac{\kappa}{\eta_{\theta}^{2}}
$$

On the other hand:

$$
\left\langle\theta^{2}\right\rangle_{D} \simeq\left\langle\theta^{2}\right\rangle\left(\frac{\eta}{\ell}\right)^{2 / 3} \simeq \frac{U_{t}^{4}}{(g \alpha \ell)^{2}}\left(\frac{\nu^{3} d}{U_{t}^{3} \ell^{4}}\right)^{1 / 6}
$$

Using equations $16,17,31$, and 32 , we have:

$$
\begin{aligned}
\beta & \simeq \frac{g \alpha d}{U_{t}^{3}}\left(\frac{U_{t}^{3}}{d \nu}\right)^{1 / 2} \frac{U_{t}^{7 / 2} \nu^{1 / 2} d^{1 / 6}}{(g \alpha \ell)^{2} \ell^{2 / 3}} \\
& =\frac{U_{t}^{2}}{g \alpha d^{2}}\left(\frac{d}{\ell}\right)^{8 / 3}
\end{aligned}
$$

and using equation 25:

$$
\begin{aligned}
\frac{N u}{\operatorname{Pr}} & =\frac{U_{t}^{3}}{d g \alpha \nu \beta} \\
& \simeq \frac{1}{d g \alpha \nu \beta}\left(g \alpha \beta d^{2}\right)^{3 / 2}\left(\frac{\ell}{d}\right)^{4} \\
& =C G r^{1 / 2}
\end{aligned}
$$

where $C=(\ell / d)^{4}$ is fixed at constant inclination angle $\psi$. This is the Hard Turbulence regime (High Prandtl Hard Turbulence HPHT).

At lower Reynolds number, $\eta$ reaches $\ell$, there is no Kolmogorov inertial range, but the velocity gradients continue to smooth down, and the temperature dissipative scale $\eta_{\theta}$ continues its growth as:

$$
\frac{\kappa}{\eta_{\theta}^{2}} \simeq\left(\frac{U_{t}^{3}}{d \nu}\right)^{1 / 2}
$$

In the absence of inertial range, $\left\langle\theta^{2}\right\rangle_{D} \simeq\left\langle\theta^{2}\right\rangle$ and, using equations 16, 17:

$$
\begin{aligned}
\beta & \simeq \frac{g \alpha d}{U_{t}^{3}} \frac{U_{t}^{4}}{(g \alpha \ell)^{2}}\left(\frac{U_{t}^{3}}{d \nu}\right)^{1 / 2} \\
& =\frac{U_{t}^{5 / 2}}{g \alpha \nu^{1 / 2} d^{3 / 2}}\left(\frac{d}{\ell}\right)^{2}
\end{aligned}
$$

As in the previous cases, we can extract the expression of $U_{t}$ versus $\beta$ :

$$
U_{t} \simeq\left(g \alpha \beta \nu^{1 / 2} d^{3 / 2}\right)^{2 / 5}\left(\frac{\ell}{d}\right)^{4 / 5}
$$


and use it in the expression of $\mathrm{Nu} / \mathrm{Pr}$ :

$$
\begin{aligned}
\frac{N u}{P r} & =\frac{U_{t}^{3}}{d g \alpha \nu \beta} \\
& \simeq\left(\frac{g \alpha \beta d^{4}}{\nu^{2}}\right)^{1 / 5}\left(\frac{\ell}{d}\right)^{12 / 5} \\
& =E G r^{1 / 5}
\end{aligned}
$$

where $E=(\ell / d)^{12 / 5}$ is fixed at constant inclination angle $\psi$. While the resulting exponent (0.2) differs from the Pawar et al. [15] one (0.3), these regimes nicely correspond in other aspects. Indeed, plotting $\mathrm{Nu} / \mathrm{Pr}$ versus $G r$, all Prandtl number merge on a single curve, as observed by Pawar et al.. Moreover, as this regime occurs between a $G r^{1 / 2}$ and a $G r^{2}$ (see below) regimes, it appears as an inflexion point, whose slope hardly reaches the asymptotic one. We shall call this regime the Batchelor regime (High Prandtl Batchelor Regime HPBR).

Finally, if the Prandtl number is not too high, in such a way that $\eta_{\theta}$ reaches $\ell[13]$ while the flow is always in a turbulent inertial regime, then $\eta_{\theta}$ cannot grow further. Then:

$$
\left\langle\partial_{i} \theta \partial_{i} \theta\right\rangle \simeq \frac{\left\langle\theta^{2}\right\rangle}{\ell^{2}}
$$

and, using equation 16 :

$$
\begin{aligned}
\beta & \simeq \frac{g \alpha d}{U_{t}^{3}} \frac{U_{t}^{4}}{(g \alpha \ell)^{2}} \frac{\kappa}{\ell^{2}} \\
& =\frac{U_{t} \kappa}{g \alpha d^{4}}\left(\frac{d}{\ell}\right)^{4}
\end{aligned}
$$

Then, using equation 25 :

$$
\begin{aligned}
\frac{N u}{P r} & =\frac{U_{t}^{3}}{d g \alpha \nu \beta} \\
& \simeq\left(\frac{g \alpha \beta d^{4}}{\kappa^{2}}\right)^{2} \frac{\kappa}{\nu}\left(\frac{\ell}{d}\right)^{12} \\
& =B^{\prime} G r^{2} \operatorname{Pr}^{3}
\end{aligned}
$$

where $B^{\prime}=(\ell / d)^{12}$ is a constant at constant inclination angle $\psi$. This is the Moderate Prandtl Soft Turbulence scaling, which can also be written $N u=B^{\prime}\left(G r P r^{2}\right)^{2}$, and is identical to the Small Prandtl Soft Turbulence $\left(B^{\prime}=B\right)$.

\section{DISCUSSION AND CONCLUSION}

Let us thus resume the succession of inertial regimes, starting from the highest Grashof numbers and going down. When the Prandtl number is low, the Hard Turbulence regime (SPHT) corresponds to the relation:

$$
N u=A\left(G r P^{2}\right)^{1 / 2}
$$

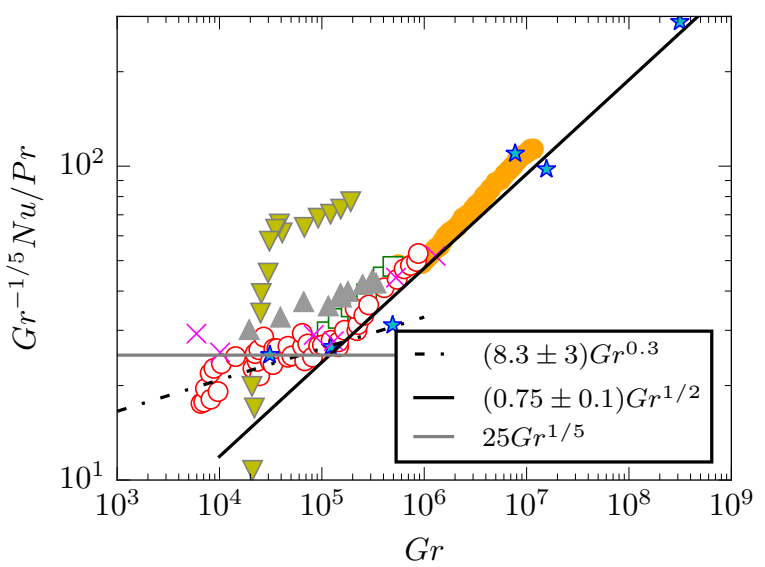

FIG. 2. Open and full circles, squares, stars and crosses are data from Pawar and Arakeri [15], corresponding to high or moderate Prandtl numbers in a circular cross section vertical channel. With this compensated plot, the visible plateau in the open circle and crosses data is the Batchelor regime. The high Gr regime is the Hard Turbulence one. The lowest Grashof open circle points probably shows the Soft Turbulence regime (moderate Prandtl). Also shown are data (gray triangles up) from reference [16], in reasonable agreement despite the square cross section of the channel. Green triangle down correspond to an inclined channel ( 5 degrees, reference $[8]$ ), and show the same succession of regimes, while with other values for the constants $A, B$, and $E$.

At lower Grashof number, but sufficient to have an inertial regime, we have the Soft Turbulence regime (SPST), where:

$$
N u=B\left(G r P r^{2}\right)^{2}
$$

The transition corresponds to the temperature dissipative scale reaching the large correlation scale [13]. On a $\mathrm{Nu}$ versus $\mathrm{Gr} \mathrm{Pr}^{2}$ diagram, all the (small) Prandtl number merge on the same curve.

When the Prandtl number is large, the highest Grashof numbers again correspond to the Hard Turbulence regime (HPHT):

$$
\frac{N u}{P r}=C G r^{1 / 2}
$$

At lower Grashof number, but sufficient to have an inertial regime, we have here the Batchelor regime (HPBR):

$$
\frac{N u}{P r}=E G r^{1 / 5}
$$

The transition corresponds to the velocity dissipative scale reaching the large correlation scale, thus to the Kolmogorov inertial range vanishing. On a $(\mathrm{Nu} / \mathrm{Pr})$ versus $G r$ diagram, all the (high) Prandtl number merge on the same curve.

At even lower Grashof number, but always sufficient to have an inertial regime (thus for moderate Prandtl numbers), we find back the Soft Turbulence regime, equation 
43. Again, the transition corresponds to the temperature dissipative scale reaching the large correlation scale [13].

We find thus all the observed regimes [8, 15], if we identify the predicted exponent for the Batchelor regime
(0.2) to the observed one [15] (0.3). Moreover, these regimes nicely underline the existence of the Kolmogorov or Batchelor ranges of scales, and their clearcut transitions.
[1] T. Seon, J.P. Hulin, D. Salin, B. Perrin, E.J. Hinch, "Laser-induced fluorescence measurements of buoyancy driven mixing in tilted tubes", Phys. Fluids, 18, 041701 (2006).

[2] J. Znaien, Y. Hallez, F. Moisy, J. Magnaudet, J.P. Hulin, D. Salin, E.J. Hinch, "Experimental and numerical investigations of flow structure and momentum transport in a turbulent buoyancy-driven flow inside a tilted tube", Phys. Fluids, 21, 115102 (2009).

[3] J. Znaien, F. Moisy, J.P. Hulin, "Flow structure and momentum transport for buoyancy driven mixing flows in long tubes at different tilt angles" , Phys. Fluids, 23, 035105 (2011).

[4] R. Delgado-Buscalioni, E. Crespo, "Flow and heat transfer regimes in inclined differentially heated cavity" , Int. Journ. of Heat and Mass transfer, 44, 1947-1962 (2001).

[5] M.Gibert, H. Pabiou, F. Chillà, B. Castaing, "HighRayleigh-Number Convection in a Vertical Channel", Phys.Rev. Letters, 96, 084501 (2006).

[6] M.Gibert, H. Pabiou, J.-C. Tisserand, B. Gertjerenken, B. Castaing, F. Chillà, "Heat Convection in a Vertical Channel: Plumes versus Turbulent Diffusion", Phys. Fluids, 21, 035109. (2009).

[7] J.C. Tisserand, M. Creyssels, M. Gibert, B. Castaing, F. Chilla, "Convection in a vertical channel", NJP 12, 075024 (2010).

[8] X. Riedinger, J.C. Tisserand, F. Seychelles, B. Castaing, F. Chillà, "Heat transport regimes in an inclined channel", Phys. Fluids, 25, 015117 (2013).

[9] E. Calzavarini, D. Lohse, F. Toschi, R. Tripiccione, "Rayleigh and Prandtl number scaling in the bulk of Rayleigh-Bénard turbulence", Phys. Fluids, 17, 055107 (2005).

[10] L.-E. Schmidt, E. Calzavarini, D. Lohse, F. Toschi, R.
Verzicco, "Axially homogeneous Rayleigh-Benard convection in a cylindrical cell", J. Fluid Mech. , 691, 52-68 (2012).

[11] M.R. Cholemari, J.H. Arakeri, "Axially homogeneous, zero mean flow buoyancy-driven turbulence in a vertical pipe", J. Fluid Mech. , 621, 69-102 (2009).

[12] D.J. Tritton, "Physical Fluid Dynamics", Oxford Science Publications, (1988)

[13] One can wonder why we do not consider the possibility of a Bolgiano range where $\left\langle\theta^{2}\right\rangle_{r}$ depends on the scale $r$ as $r^{2 / 5}$, down to a Bolgiano scale $\ell_{B}$. Such a range has often be reported in convection flows $[17,18]$. However, the corresponding velocity structure function behavior (scaling as $r^{6 / 5}$ ) has never been observed. Moreover, $\ell_{B}$ should be of order $\ell$, and it is never envisaged that $\eta_{\theta}$ be smaller than $\ell_{B}$. Thus, the limit of the Soft Turbulence could simply correspond to $\eta_{\theta}=\ell_{B}$ rather than $\ell$, without any change in the conclusions.

[14] H. Tennekes and J. Lumley, "A first course in Turbulence", MIT press, Cambridge, Massachusetts, and London, England (1972).

[15] S.S. Pawar and J.H. Arakeri, "Two regimes of flux scaling in axially homogeneous turbulent convection in vertical tube", Phys. Rev E (2016).

[16] Échanges turbulents en convection thermique, Ph.D. Thesis, ENS de Lyon (2014)

[17] F. Chillà, S. Ciliberto, C. Innocenti, E. Pampaloni, "Spectra of local and averaged scalar fields in turbulence" EPL 22, 23-28 (1993).

[18] S.S. Pawar and J.H. Arakeri, "Kinetic energy and scalar spectra in high Rayleigh number axially homogeneous buoyancy driven turbulence", Phys. Fluids, 28, 065103 (2016). 